\title{
Border Shape
}

National Cancer Institute

\section{Source}

National Cancer Institute. Border Shape. NCI Thesaurus. Code C69224.

A description of the boundary configuration of a sample. 